\title{
Sustentabilidade socioambiental: um estudo bibliométrico da evolução do conceito na área de gestão de operações
}

\author{
Gustavo Menoncin de Carvalho Pereira**, Chen Yen-Tsang ${ }^{\mathrm{b}}$, \\ Reinaldo Belickas Manzinic, Nádia Vernes Almeida ${ }^{\mathrm{d}}$ \\ a*gustavo.pereira@gvmail.br, EAESP, Brasil \\ byentsang.chen@gmail.com, EAESP, Brasil \\ crbmanzini@gmail.com, EAESP, Brasil \\ dnadiavalmeida@gmail.com, EAESP, Brasil
}

\begin{abstract}
Resumo
A Sustentabilidade Socioambiental (SS) apresenta desafios para organizações, governos e sociedade, tanto para a compreensão de seus conceitos teóricos quanto para a transformação de seus pressupostos em práticas operacionais e administrativas. Pesquisadores e organizações têm procurado conceitos, modelos e ferramentas, num esforço de adaptação ao novo paradigma, inclusive Gestão de Operações (G0). Neste artigo, é apresentada uma pesquisa bibliométrica, com o objetivo de investigar a presença do tema SS nos artigos do International Journal of Operations \& Production Management e traçar um paralelo da pesquisa realizada com artigos publicados em periódicos brasileiros relevantes de GO. Os resultados deste trabalho apontam a evolução de SS em GO e identificam os principais temas abordados em SS. A principal conclusão é de que as cocitações envolvendo SS vêm crescendo em G0, posicionando o tema próximo à discussão de Estratégia de Manufatura.
\end{abstract}

Palavras-chave

Sustentabilidade socioambiental. Gestão de operações. Estudo bibliométrico. Visão baseada em sustentabilidade. Cadeia de suprimento verde.

\section{Introdução}

O tema Sustentabilidade Socioambiental (SS) adquire especial importância à medida que a preservação do meio ambiente torna-se cada vez mais um tema de grande relevância para a sociedade como um todo, gerando discussões e polêmicas que envolvem, principalmente, empresários, formuladores de políticas e acadêmicos (OLIVEIRA; BORGES; JABBOUR, 2005). SS é um conceito que está relacionado a um novo paradigma, o desenvolvimento sustentável, o qual foi definido no relatório "Nosso futuro comum", fruto da Comissão Mundial sobre Meio Ambiente e Desenvolvimento 1983-1987, como

[...] aquele que atende às necessidades do presente sem comprometer a possibilidade das gerações futuras de atenderem as suas próprias necessidades. (COMISSÃO MUNDIAL SOBRE MEIO AMBIENTE E DESENVOLVIMENTO - CMMAD, 1988 apud BARBIERI, 1997, p. 23).
0 esgotamento de recursos naturais, a poluição do solo e águas subterrâneas por fontes industriais, os acidentes nucleares e químicos (Three Mile lsland, Chernobyl, Bhopal, Seveso, Basileia, Alasca, Baía de Guanabara, entre outros), os desastres provocados pelo homem (o encolhimento do Mar de Aral, a destruição ambiental na Europa do Leste), o aquecimento global da atmosfera e o efeito estufa, passaram a representar um verdadeiro desafio para a sociedade e para as empresas (SACHS, 1993; SEIFFERT, 2008; EPELBAUM, 2006). Segundo Barbieri (2007), o grande desafio para as empresas é a adaptação de sua gestão às demandas do meio ambiente. As preocupações ambientais não surgem espontaneamente, são influenciadas por três grandes conjuntos de forças que interagem reciprocamente: governo, sociedade e mercado. Simon (2003) seleciona cinco dilemas fundamentais (o que chama de dilemas-chave) que 
estão no centro dos debates sobre teoria, política e praxis do desenvolvimento sustentável: 1) tradução do conceito de "desenvolvimento", da teoria para a prática; 2) mudança do paradigma e a implantação de alternativas radicais (não permitir a burocratização do paradigma); 3) o global no local, e vice-versa: a dialética da escala; 4) o jogo do trade-off: ampliação de horizontes, contenção de custos e promoção da sustentabilidade; 5) aproveitamento da tecnologia.

Inicialmente, num movimento que pode ser denominado de "adaptação resistente", as empresas adotaram medidas para a eliminação de danos, impulsionadas por legislação decorrente de acordos internacionais e políticas públicas (VILELA JUNIOR; DEMAJOROVIC, 2006). Ainda na entrada do século XXI, estudos como o de Schaefer e Harvey (2000), e Hunt e Raman (2000) demonstram que a gestão ambiental nas empresas é fortemente influenciada pela regulação direta, e que estas tendem a definir sua responsabilidade da mesma maneira que essas questões são reguladas no seu segmento industrial.

Segundo Schaefer (2009), há um longo debate sobre as relações entre a regulação ambiental e a performance econômica das empresas. Com referência às iniciativas de autorregulação, embora funcionem sob determinadas circunstâncias, não parecem funcionar em todas as situações. Porter e van der Linde (1995), Pickman (1998) e King (2000) entendem que a regulação ambiental pode estimular a inovação tecnológica e institucional. Para outros autores, Smith e Crotty (2006), por exemplo, a regulação ambiental não induz automaticamente as empresas à inovação.

Para efetivar qualquer abordagem, as empresas devem realizar atividades administrativas e operacionais, com base em modelos, entendidos como construções conceituais para alcançar objetivos definidos. Barbieri (2007) cita como modelos: programa de atuação responsável, administração da qualidade ambiental total (TQEM), produção mais limpa $(\mathrm{P}+\mathrm{L})$, ecoeficiência, projeto para o meio ambiente (design for environment ou ecodesign), metabolismo industrial, ecologia industrial, simbiose industrial. A adoção de qualquer modelo de gestão ambiental requer o uso de instrumentos (meios ou ferramentas) para que as empresas possam alcançar objetivos específicos. Enumeram-se aqui: auditoria ambiental, avaliação do ciclo de vida, estudos de impactos ambientais, sistemas de gestão ambiental, relatórios ambientais, rotulagem ambiental, gerenciamento de riscos ambientais, educação ambiental empresarial. Destacam-se as normas relativas aos sistemas de gestão ambiental produzidas pela ISO (International Organization for Standardization).

Desde a década de 1990, as empresas que estão na vanguarda da gestão ambiental têm transformado desafios em novas oportunidades, buscando conceitos, modelos e instrumentos (ferramentas) para tornar efetivos os seus programas de gestão ambiental. Os autores (VILELA JUNIOR; DEMAJOROVICK, 2006) fazem uma distinção entre as ferramentas amplamente aceitas, como produção mais limpa, sistemas de gestão ambiental, auditorias ambientais, gerenciamento de risco, ecoeficiência, avaliação de impacto ambiental, educação ambiental e gerenciamento de passivos ambientais, e as que mais recentemente começaram a ser incorporadas pelas empresas, tais como: análise do ciclo de vida, ecodesign, rotulagem ambiental e outras.

Höjer et al. (2008) reconhecem que a integração dos aspectos ambientais no planejamento das organizações exige o desenvolvimento de ferramentas poderosas e eficientes para tornar possível a compreensão e avaliação dos sistemas econômicos, tecnológicos e ambientais, e também para possibilitar a essas organizações a formação de uma estrutura eficiente de informações, necessária à alimentação dos seus processos de tomada de decisões.

Com relação à estratégia nas empresas, Silva e Santos (2006) distinguem: estratégia da corporação, da unidade de negócio e da produção. A área de gestão da produção usa as diretrizes de estratégia corporativa, adaptando-as às suas metodologias. As principais abordagens estratégicas corporativas são: estratégias competitivas de Porter (1980) e a Visão Baseada em Recursos (RBV), que foi impulsionada por Wernerfelt (1984); Barney (1991) e Grant (1991).

Para Kleindorfer, Singhal e Wassenhove (2005), em gestão de operações, tanto pesquisadores quanto organizações têm enfrentado novos desafios para atenderem a uma nova realidade de ordem social e ambiental. A evolução em direção à Gestão de Operações Sustentável envolve três áreas: produtos verdes e desenvolvimento de processos; gestão de operações "Lean and Green", Remanufatura; e Closed-loop supply chains.

Ao analisarmos os aspectos centrais das discussões em torno da Estratégia de Operações (EO) tratadas em GO, no mundo acadêmico, temos: a) a consistência entre estratégia de negócios e de operações (Manufacturing - missing link in corporate strategy) (SKINNER, 1969); b) os quatro estágios de competitividade da área de operações (Restoring our Competitive Edge: Competing Through) (HAYES; WHEELWRIGHT, 1984); c) a taxonomia das estratégias de manufatura ( $A$ Taxonomy of Manufacturing Strategies) (MILLER; ROTH, 1994); d) a necessidade da produção de estudos empíricos de modo a fortalecer a construção de uma teoria para a EO (Manufacturing strategy, environmental uncertainty and performance: a path and analytic 
model) (SWAMIDASS; NEWELL, 1987); e e) o modelo cumulativo de competências ("cone de areia”) (Lasting improvements in manufacturing performance: in search of a new theory) (FERDOWS; DE MEYER, 1990). Nenhum dos artigos centrais sobre EO aponta a SS como fator a ser incorporado na formulação de suas estratégias, com o enfoque sempre voltado à utilização dos recursos de acordo com requisitos das prioridades competitivas (qualidade, custo, entrega, flexibilidade), e visando, igualmente, a uma maior aproximação com mercados consumidores.

Para tentar responder à questão formulada no início deste texto, a pesquisa propõe fazer a ampliação do estudo de Pilkington e Fitzgerald (2006), agregando o período de 2004 a 2008, e estabelece como objeto de investigação a presença do tema SS nos artigos do 1JOPM. Estes autores utilizaram como base as publicações do International Journal of Operations \& Production Management (IJOPM), no período compreendido entre os anos de 1994 a 2003, e os resultados da investigação detectaram nos trabalhos publicados no IJOPM, que o tema SS surge apenas em período recente (1999-2003)

Com o objetivo de se traçar um paralelo com a produção de periódicos brasileiros da área de G0, recorreu-se a um estudo qualitativo, o qual analisou os artigos publicados nos dois periódicos brasileiros de maior relevância em GO. Foram selecionados os artigos que abordavam o tema SS e, através de sua leitura, foi realizado um agrupamento por afinidade. 0 objetivo é entender se, no mesmo período que o tema ganhou uma maior importância no IJOPM, tal evento ocorre de maneira paralela no Brasil.

A presente pesquisa tem também a intenção de contribuir para a formação de um panorama sobre a produção científica que envolve o tema SS na área de GO, no período de 1994 a 2008, bem como disseminar a metodologia da pesquisa bibliométrica. Espera-se que seus resultados indiquem temas para pesquisas futuras, tendo como fonte de dados outros importantes periódicos da área.

\section{Revisão teórica}

A Bibliometria, através da utilização de análises estatísticas, mede a produção de pesquisa científica e tecnológica, na forma de artigos, publicações, citações, patentes e outros indicadores mais complexos, o que permite avaliar atividades de pesquisa, laboratórios, cientistas, instituições, países etc. Nas análises, utiliza-se a comunicação formal entre os cientistas - artigos, livros, patentes, documentos -, e não são consideradas as comunicações informais, tais como: oral, relatórios, conferências, comunicação eletrônica não científica. Apesar de limitações, a bibliometria proporciona uma medida quantitativa essencialmente objetiva da produção científica (OKUBO, 1997).

Neste tópico, são apresentados os fundamentos do estudo bibliométrico de autores que produziram artigos sobre temas relacionados à GO (RAMOSRODRIGUES; RUIZ-NAVARRO, 2004; PILKINGTON; FITZGERALD, 2006; PILKINGTON; MEREDITH, 2009), através da construção da matriz de cocitações e da Análise fatorial.

\subsection{A construção da matriz de cocitações}

De acordo com Small (1973), a cocitação pode ser definida como sendo a frequência com a qual dois trabalhos prévios de uma determinada área literária são citados conjuntamente por trabalhos posteriores. A "força" da cocitação é mensurada a partir do nível de relacionamento ou associação entre artigos dados pela população de autores citados. Os padrões de cocitações se alteram à medida que os interesses e padrões intelectuais de uma determinada área mudam. Pode-se assumir que artigos frequentemente citados representam os conceitos, métodos ou experimentoschave de uma área de pesquisa. Neste sentido, os padrões de cocitação podem ser utilizados para mapear, em detalhe, os relacionamentos entre estas ideias, ou seja, se trata de um modo objetivo de modelar a estrutura intelectual de determinado campo científico.

De modo a identificar padrões de comportamento mais detalhados, Pilkington e Fitzgerald (2006) utilizaram como unidade de análise o artigo, revelando a "força" individual de cada um deles ao longo do tempo.

\subsection{Análise fatorial}

A análise fatorial é um método de análise de interdependências que permite criar construtos $r$ que agrupam variáveis interdependentes $v$, no qual $r<v$. Emprega-se a análise fatorial para se reduzir um conjunto de 10, 20, 30 ou mais variáveis em 3, 4, 5 (ou mais) dimensões ou fatores sem perder a capacidade explicativa. Os fatores são entidades (construtos) hipotéticas não diretamente observáveis, que se inferem ou se constroem a partir do agrupamento das variáveis. A análise fatorial parte da análise das correlações entre certo número de variáveis, permitindo a definição de dimensões subjacentes chamadas fatores. Estes fatores podem ser empregados para a redução e o agrupamento das variáveis inicialmente computadas (HAIR JUNIOR, 2005). Variáveis que apresentam elevada interdependência são medidas de um mesmo fenômeno, podendo ser agrupadas em uma 
variável composta a partir de combinações lineares das variáveis iniciais. Seu propósito é o de expressar a matriz de covariância de um determinado conjunto de variáveis observadas em termos de um conjunto menor de variáveis implícitas, não observáveis diretamente. Sua utilidade como instrumento de pesquisa está justamente na capacidade de detectar relações entre as variáveis, e através de análise qualitativa adequada pode levar à reconstrução dos conceitos que deram origem às variáveis iniciais.

\section{Metodologia e amostra}

A pesquisa seguiu uma sequência de etapas, conforme apresentado na Figura 1. As etapas envolveram os critérios de seleção dos periódicos, o acesso aos artigos, a atualização do trabalho de Pilkington e Fitzgerald (2006), análise dos artigos e apresentação dos resultados.

\subsection{Seleção de periódicos: sustentabilidade em gestão de operações}

A primeira etapa buscou identificar publicações da área de G0 para a pesquisa do tema Sustentabilidade, selecionando o IJOPM, como periódico internacional, e as Revistas Gestão \& Produção e Produção, como periódicos nacionais.

A escolha do IJOPM, além de manter a referência de Pilkington e Fitzgerald (2006), é justificada, segundo Charvet et al. (2008), por ser o quinto journal com mais citações contendo temas de GO, como a Gestão da Cadeia de Suprimentos (Supply Chain Management) e possuir como fonte o Business Source Complete. Goh et al. (1997) classificam este journal como o terceiro mais relevante na área, dentre 48 analisados. O IJOPM começou a ser publicado em 1980, com mais de 1.600 artigos publicados na área.

Os periódicos brasileiros selecionados foram revistas brasileiras da área de Administração que têm conceito mais alto no QUALIS-CAPES (WEBQUALIS), e que tratam de G0. A revista Gestão e Produção e a revista Produção foram as selecionadas.

\subsection{Coleta dos artigos}

Pilkington e Fitzgerald (2006) identificaram as ideias centrais da área de G0 no período compreendido entre 1994 e 2003. Iniciaram a partir de 1994, ano em que todos os artigos do journal estão disponíveis na íntegra e eletronicamente. Portanto, o primeiro período compreende os anos de 1994 a 1998, e o segundo, os anos de 1999 a 2003. Neste trabalho, a matriz de cocitações gerada permitiu que, através da aplicação da técnica de Análise Fatorial, se revelasse o agrupamento de autores por tema. Cada um dos temas foi nomeado a partir da convergência de seu conteúdo, apresentado no Quadro 1.

Os artigos centrais do período de 1999 a 2003, sob o tema Visão Baseada em Sustentabilidade, são dois: A Natural-Resource-Based View of the Firm (HART, 1995) e Green and Competitive: Ending the Stalemate (PORTER; VAN DER LINDE, 1995).

A pesquisa realizada na base de dados de artigos publicados na Gestão e Produção envolveu 203 artigos publicados de 2004 a 2008. Foram identificados 20 artigos com temas próximos a SS. No caso da revista Produção, dos 182 artigos, no mesmo período, 10 abordaram temas de SS. A pesquisa foi viabilizada

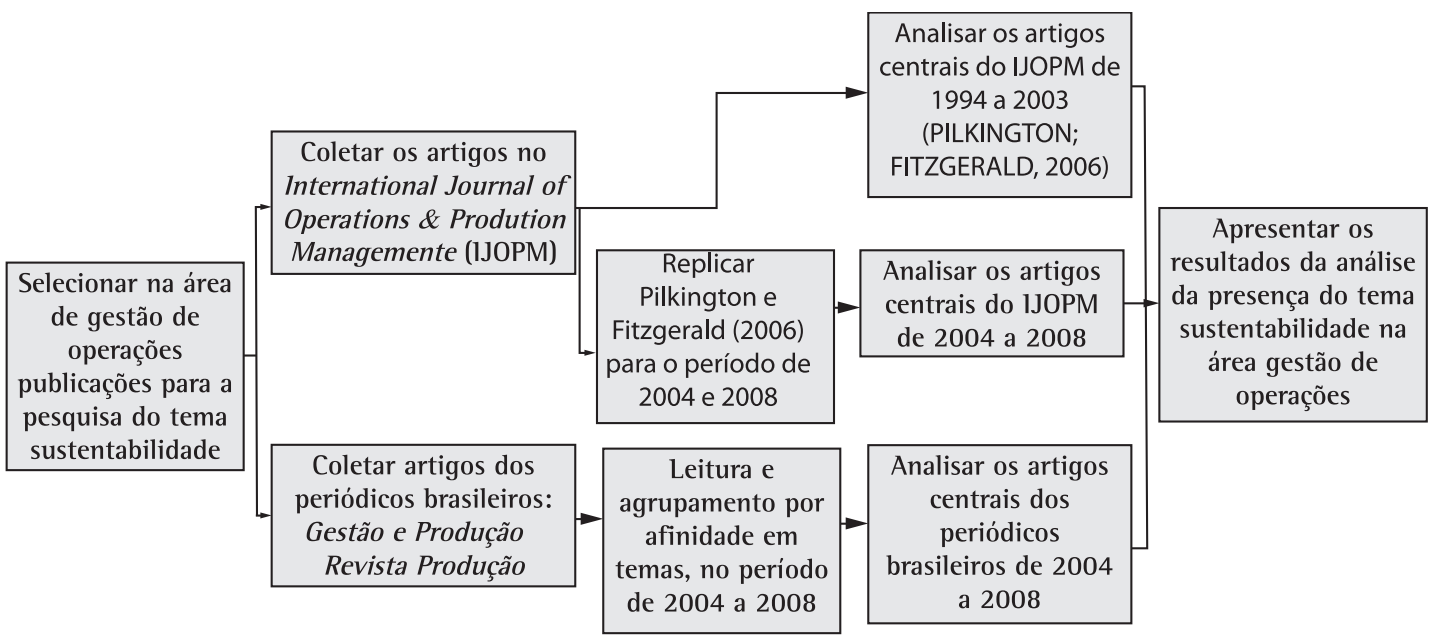

Figura 1. Etapas da metodologia. Fonte: Os autores. 
Quadro 1. Temas identificados em gestão de operações.

\begin{tabular}{|c|c|}
\hline 1994-1998 & 1999-2003 \\
\hline Estratégia de manufatura & Estratégia de manufatura \\
\hline Sistema Japonês de Manufatura & Visão Baseada em Recursos (RBV) \\
\hline Processos e Reengenharia (BPR) & Indicadores: BSC \\
\hline Indicadores & $\begin{array}{l}\text { Construção de teoria e } \\
\text { análise quantitativa }\end{array}$ \\
\hline Tempo (vantagem competitiva) & Sistemas Lean \\
\hline Teoria e métodos de pesquisa & $\begin{array}{l}\text { Métodos qualitativos e estudo } \\
\text { de caso }\end{array}$ \\
\hline Disseminação da técnica JIT & $\begin{array}{l}\text { Logística e suprimento } \\
\text { estratégicos (SCM) }\end{array}$ \\
\hline Engenharia simultânea & Controle e projeto de processos \\
\hline Simulação & Visão baseada em sustentabilidade \\
\hline Planejamento de estoques e MRP & \\
\hline
\end{tabular}

através da consulta à base Scielo de ambas as revistas, disponíveis com os artigos na íntegra para leitura e análise.

\subsection{Atualização do artigo de Pilkington e Fitzgerald (2006)}

A fim de garantir a comparabilidade entre os resultados, este trabalho replicou a metodologia empregada por Pilkington e Fitzgerald (2006), excetuando-se os softwares aplicativos para a manipulação da base de dados e análise estatística. Os autores empregaram a ferramenta Bibexcel e SPSS, e esta pesquisa empregou a ferramenta Sitkis e Ucinet. Este aplicativo importa registros da base de dados ISI Web of Science criando uma base de dados no aplicativo Microsoft Access, que pode ser manipulada (SCHILDT, 2002). As análises posteriores foram construídas através do software Ucinet (BORGATTI; EVERETT; FREEMAN, 2002), destinado à análise de redes sociais.

Para construir a matriz de cocitações referente ao período de 2004 a 2008, a base de dados empregada foi a mesma utilizada por Pilkington e Fitzgerald (2006). No período compreendido entre os anos de 2004 a 2008 do IJOPM, isto é, desde a edição número 11, volume 23 (2003), até a edição do número 9, volume 28 (2008), foram recuperados 339 artigos, perfazendo um total de 756 autores e 11.765 citações, das quais foram descartadas 1.254 (ou 10,65\%), por estarem danificadas. Na geração da matriz de cocitações, empregaram-se, como limite de corte, artigos ou livros com no mínimo sete citações, aproximadamente metade do empregado por Pilkington e Fitzgerald (2006), cuja amostra e horizonte de tempo eram duas vezes maiores. 0 número de observações geradas foi de 86 registros, ou seja, obteve-se uma matriz quadrada de 86 colunas por 86 linhas conforme.
No caso, a análise fatorial foi realizada a partir da matriz de cocitações original. 0 método utilizado foi o dos Componentes Principais com rotação varimax.

Para os artigos publicados nos periódicos nacionais, os artigos foram lidos e agrupados por afinidade em temas que se assemelham àqueles abordados pelos artigos do IJOPM.

\section{Resultados}

\subsection{Ampliação da base de dados de citações e cocitações do IJOPM (2004-2008)}

Como resultado da aplicação da Análise Fatorial, oito fatores foram encontrados e nomeados a partir da leitura dos abstracts e, em alguns casos, de todo o conteúdo dos artigos, formando assim os temas centrais de discussão no IJOPM neste período de 2004 a 2008. 0 Quadro 2 apresenta os tópicos, autores centrais e periféricos a cada um.

Com a ampliação da base do estudo pela adição do período de 2004 até 2008, quatro artigos passam a ser centrais para o tema: Regional Creative Destruction: Production Organization, Globalization, and the Economic Transformation) of the Midwest (FLORIDA, 1996); Suppliers and Environmental Innovation: The Automotive Paint Process (GEFFEN; ROTHENBERG, 2000); Does it Really Pay to be Green? An Empirical Study of Firm Environmental and Financial Performance (KING; LENOX, 2001) e Green Purchasing Strategies: Trends and Implications (MIN; GALLE, 1997).

\subsection{Temas em GO: comparação entre períodos}

0 Quadro 3 apresenta, em ordem decrescente de cocitações, uma comparação dos temas identificados em GO, entre os períodos analisados por Pilkington e Fitzgerald (1994-1998; 1999-2003) e o de 2004-2008.

Ao se comparar os temas identificados por Pilkington e Fitzgerald (2006) para os períodos de 1994-1999 e 2000-2003 ante o período 2004-2008, observa-se que o tema estratégia de manufatura perde importância. Da primeira posição ocupada nos períodos anteriores, ele passa para a quinta no último período, passando a ser citado com menos frequência do que temas tais como Alianças e Parcerias, Sistemas Flexíveis de Produção (Lean Manufacturing) e Gestão da Cadeia de Suprimentos (Supply Chain Management). Ao compararmos os autores centrais que servem de referência para a produção de artigos neste tema, houve pouca evolução. Os mesmos autores centrais 
Quadro 2. Temas e artigos/autores vinculados.

\begin{tabular}{|c|c|}
\hline Tema & Artigos/Autores \\
\hline $\begin{array}{l}\text { Visão baseada em recursos } \\
\text { (Resource based view) }\end{array}$ & $\begin{array}{l}\text { Barney (1991); Eisenhardt (2000); Hamel (1994); Peteraf (1993); Prahalad (1990), Stalk (1992); Teece } \\
\text { (1997); Womack (1994) }\end{array}$ \\
\hline $\begin{array}{l}\text { Alianças / redes } \\
\text { (Alliances/network) }\end{array}$ & $\begin{array}{l}\text { Bollen (1989); Carr (1999); Chen (2004); Cousins (1999); Dyer (1998); Ford (1997); Fornell (1981); } \\
\text { Frohlich (2001); Handfield (2002); Harland (1996, 1999); Krause (1998); Lamming (1993); McCutcheon } \\
\text { (2000); Mohr (1994); Nishiguchi (1994); Porter (1985); Ring (1992); Williamson (1975); Womack (1990) }\end{array}$ \\
\hline $\begin{array}{l}\text { Sistemas flexíveis de produção } \\
\text { (Flexible production systems) }\end{array}$ & $\begin{array}{l}\text { Beamon (1999); Detoni (1998); Gerwin (1987, 1993); Gupta }(1989,1992) \text {; Mason-Jones (1999); Sethi } \\
\text { (1990); Slack (1987); Upton (1994) }\end{array}$ \\
\hline $\begin{array}{l}\text { Gestão da cadeia de suprimentos } \\
\text { (Supply chain mgmt) }\end{array}$ & $\begin{array}{l}\text { Chandrashekar (1999); Cooper (1997); Cox (2001); Ellram (1991); Giannakis (2004); Handfield (1999); } \\
\text { Stank (2001); Waller (2000) }\end{array}$ \\
\hline $\begin{array}{l}\text { Estratégia de manufatura } \\
\text { (Manufacturing strategy) }\end{array}$ & $\begin{array}{l}\text { Ferdows; De Meyer (1990), Flynn, Schroeder e Flynn (1999); Hayes e Wheelwright (1979); Hayes (1984); } \\
\text { Leong, Snyder e Ward (1990); Miller e Roth (1994); Nunnally (1978); Schonberger (1986); Skinner } \\
\text { (1969), Smith e Reece (1999); Swamidass e Newell (1987); Venkatraman (1986) }\end{array}$ \\
\hline $\begin{array}{l}\text { Cadeia de suprimentos "verde" } \\
\text { ("Green" supply chain) }\end{array}$ & Florida (1996); Geffen (2000); King (2001); Min (1997) \\
\hline Aglomerações (Aglomeration) & Harrigan (1985); Williamson (1985) \\
\hline $\begin{array}{l}\text { Medição de indicadores de desempenho } \\
\text { (Performance/measures) }\end{array}$ & Dixon (1990); Fitzgerald (1991); Hayes (1980); Johnson (1987); Stalk (1988) \\
\hline
\end{tabular}

Fonte: Os autores.

Quadro 3. Temas identificados em gestão de operações.

\begin{tabular}{|c|c|c|c|c|c|}
\hline \multicolumn{2}{|r|}{ 1994-1998 } & \multicolumn{2}{|r|}{ 1999-2003 } & \multicolumn{2}{|r|}{ 2004-2008 } \\
\hline 1 & Estratégia de manufatura & 1 & Estratégia de manufatura & 1 & Visão baseada em recursos \\
\hline 2 & Sistema japonês de manufatura & 2 & Visão baseada em recursos & 2 & Alianças e redes \\
\hline 3 & Processos e reengenharia (BPR) & 3 & Indicadores : BSC & 3 & Sistemas flexíveis de produção \\
\hline 4 & Indicadores & 4 & Construção de teoria e análise quantitativa & 4 & Gestão da cadeia de suprimentos \\
\hline 5 & Tempo como vantagem competitiva & 5 & Sistemas Lean & 5 & Estratégia de manufatura \\
\hline 6 & Teoria e seleção de métodos de pesquisa & 6 & Métodos qualitativos e estudo de caso & 6 & Cadeia de suprimentos "verde" \\
\hline 7 & Disseminação da técnica JIT & 7 & Gestão da cadeia de suprimentos & 7 & Aglomerações \\
\hline 8 & Engenharia simultânea & 8 & Controle e projeto de processos & 8 & Medição de indicadores de desempenho \\
\hline 9 & Simulação & 9 & Visão baseada em sustentabilidade & & \\
\hline 10 & Planejamento de estoques e MRP & & & & \\
\hline
\end{tabular}

dos dois primeiros períodos continuam sendo os mais representativos e, com a adição do terceiro período, o artigo central mais recente é de 1994.

De segundo tema mais representativo em G0 em 1999-2003, a Visão Baseada em Recursos (Resource Based View - RBV passa a ser o primeiro.

0 tema Sustentabilidade Socioambiental evolui de última posição, no período de 1999 a 2003, para o sexto, devido, talvez, à pressão dos movimentos sociais em torno da questão. 0 tema Aglomerações completa este conjunto de temas emergentes em GO.

\subsection{Temas identificados em sustentabilidade socioambiental}

A atualização da base identificada por Pilkington e Fitzgerald (2006) revela que o tema Sustentabilidade Socioambiental se mantém como um subcampo de análise dentro de $\mathrm{GO}$, mas tem sua importância relativa aumentada, passando a ocupar a sexta posição.
0 Quadro 4 apresenta o sumário de expressões escolhidas dos artigos que apareceram no resultado da pesquisa como os mais lidos. Partiu-se de expressões selecionadas através da leitura dos artigos, e consideradas representativas dos temas neles tratados. Há expressões que foram mantidas no idioma inglês, devido ao seu uso frequente em administração. Percebe-se que os seis textos foram publicados entre 1996 e 2001 e, à exceção do artigo de Florida (1996), todos apresentaram como tema central questões de investigação relacionadas ao meio ambiente. Nota-se que, de uma maneira geral, os temas tratados nos artigos concentram-se nas áreas de estratégia empresarial, regulação governamental, meio ambiente e sistemas de gestão ambiental, economia, inovação, parcerias/redes/cooperação. A leitura dos artigos permite concluir que eles tratam de temas estruturais para a formação de novas teorias, o que pode justificar o resultado da pesquisa. Ao apresentar os resultados que se referem ao período 
Quadro 4. Artigos Centrais de Sustentabilidade em GO (1994 a 2008) no IJOPM.

\begin{tabular}{|c|c|c|c|}
\hline Período & Título & Proposição de pesquisa/artigo & Sumário de expressões presentes nos artigos \\
\hline \multirow[t]{2}{*}{ 1998-2003 } & $\begin{array}{l}\text { A Natural Resource } \\
\text { View of the Firm } \\
\text { (HART, 1995) }\end{array}$ & $\begin{array}{l}\text { Dar enfoque ambiental à teoria dos } \\
\text { recursos da firma (fator "natureza"). }\end{array}$ & $\begin{array}{l}\text { Teoria dos Recursos da Firma; vantagem competitiva; } \\
\text { cooperação tecnológica; atuação responsável; prevenção } \\
\text { da poluição. }\end{array}$ \\
\hline & $\begin{array}{l}\text { Green and Competitive: } \\
\text { Ending the Stalemate } \\
\text { (PORTER; LINDE, 1995) }\end{array}$ & $\begin{array}{l}\text { Discorrer sobre os trade-offs, entre } \\
\text { produtividade, inovação, meio } \\
\text { ambiente e competitividade. }\end{array}$ & $\begin{array}{l}\text { Trade-off; competitividade; regulação ambiental; } \\
\text { sistema closed-loop; prevenção e custos de oportunidade } \\
\text { (poluição). }\end{array}$ \\
\hline \multirow[t]{4}{*}{ 2004-2008 } & $\begin{array}{l}\text { Regional Creative Destruction: } \\
\text { Production Organization, } \\
\text { Globalization, and the Economic } \\
\text { Transformation of the Midwest } \\
\text { (FLORIDA, 1996) }\end{array}$ & $\begin{array}{l}\text { Examinar como novas formas de } \\
\text { organização da produção podem } \\
\text { impactar a transformação regional } \\
\text { econômica. }\end{array}$ & $\begin{array}{l}\text { Destruição criativa; novas formas de organização } \\
\text { da produção; desinvestimento; desindustrialização; } \\
\text { globalização; geografia econômica; integração de } \\
\text { fornecedores no desenvolvimento de produto; ciclo } \\
\text { virtuoso de imitação, adaptação e aprimoramento. }\end{array}$ \\
\hline & $\begin{array}{l}\text { Suppliers and Environmental } \\
\text { Innovation: The } \\
\text { Automotive Paint Process } \\
\text { (GEFFEN; ROTHENBERG, 2000) }\end{array}$ & $\begin{array}{l}\text { Examinar as parcerias entre } \\
\text { fabricantes e fornecedores do } \\
\text { ponto de vista da melhora de } \\
\text { performance ambiental. }\end{array}$ & $\begin{array}{l}\text { Melhoria de processos; gestão ambiental; rede de } \\
\text { fornecedores; custo de compliance; prevenção da } \\
\text { poluição; design for environment; conhecimento } \\
\text { organizacional. }\end{array}$ \\
\hline & $\begin{array}{l}\text { Does it Really Pay to be Green? } \\
\text { An Empirical Study of } \\
\text { Firm Environmental and } \\
\text { Financial Performance } \\
\text { (KING; LENOX, 2001) }\end{array}$ & $\begin{array}{l}\text { Investigar a relação entre lucro e } \\
\text { desempenho ambiental e a origem } \\
\text { desta relação. }\end{array}$ & $\begin{array}{l}\text { Emissões industriais; estratégias win-win; hipóteses pays } \\
\text { to be green; regulação ambiental; cleaner industries; } \\
\text { alta performance ambiental; posicionamento da firma } \\
\text { na indústria. }\end{array}$ \\
\hline & $\begin{array}{l}\text { Green Purchasing Strategies: } \\
\text { Trends and Implications } \\
\text { (MIN; GALE, 1997) }\end{array}$ & $\begin{array}{l}\text { Apresentar resultados de pesquisa } \\
\text { (Associação de compradores EUA) } \\
\text { em empresas que realizam compras } \\
\text { verdes. }\end{array}$ & $\begin{array}{l}\text { Resíduos sólidos; embalagens verdes; reciclagem e reuso } \\
\text { de embalagens; supply chain; normas e certificações } \\
\text { ambientais; estratégia de compras verdes; regulação } \\
\text { ambiental; parceria entre compradores e fornecedores. }\end{array}$ \\
\hline
\end{tabular}

Fonte: os autores.

de 1998 a 2008, é possível afirmar que ainda é grande o interesse por textos do período entre 1996 e 2001. Pode-se avaliar a conscientização ambiental empresarial à luz de diversos estágios evolutivos, que se constituem a partir da proposição de importantes autores e que formam uma grande pluralidade de níveis de maturidade (JABBOUR; SANTOS, 2006).

Analisando o universo dos seis artigos centrais em SS no IJOPM, podemos verificar duas linhas de pensamento que se iniciaram no primeiro período em que o tema passou a ser relevante (1999-2003). 0 artigo central de Porter e Linde (1995) relaciona-se com Estratégia; o de Hart (1995), com a visão baseada em recursos. Ambos os temas representam, no período de 1994 a 2008, os mais citados no IJOPM.

Porter e Linde (1995), autores de um dos dois artigos centrais do primeiro período, iniciaram a discussão dos trade-offs, que Skinner (1969) já apresentava quando da formulação da Estratégia de Operações (EO) a ser desenvolvida pelas firmas. E0 foi o principal tema de G0 de 1994 a 2003, tendo Skinner como central nesta discussão. No seu artigo de 1995, Porter e Linde referem-se à inclusão do meio ambiente como variável a ser analisada e priorizada pelas firmas, somando-se à produtividade, inovação e competitividade. King e Lenox (2001) enriquecem o tópico, relacionando o lucro com o desempenho ambiental das firmas. Interessante notar que no período mais recente (2004-2008) SS e E0 estão muito próximas em termos de citações no IJOPM.
Hart (1995), em outro artigo central do primeiro período, propõe uma extensão da teoria de RBV. Considera a gestão do fator "natureza" como um recurso que pode vir a gerar vantagem competitiva para a firma. A evolução da discussão desta proposta em G0 é a revisão da organização da produção e seu impacto na economia regional (FLORIDA, 1996), com a integração de fornecedores e fabricantes para obter melhor performance ambiental (GEFFEN; ROTHENBERG, 2000), incluindo a áreas de compras de materiais e serviços com o conceito verde (MIN; GALLE, 1997). A RBV atualmente é o tema com maior numero de citações no IJOPM, e os artigos centrais, de 1999 a 2008, passam a tratar de temas relacionados à gestão ambiental como indutora de vantagem competitiva para a firma.

\subsection{Temas identificados nos periódicos brasileiros}

Nos periódicos brasileiros pesquisados, os 20 artigos da Gestão e Produção e os 10 artigos da Produção abordaram temas que podem ser agrupados, por afinidade, em quatro grupos: Gestão Ambiental, Sustentabilidade, Meio Ambiente e Desenvolvimento Sustentável. A diferença na quantidade de artigos de um periódico para o outro se deve ao fato de que o volume 3 de 2006 da revista Gestão e Produção foi dedicado exclusivamente ao tema. No editorial deste volume, é enfatizada a crescente preocupação 
Tabela 1. Agrupamento de Temas abordados nos periódicos brasileiros próximos a SS (2004 a 2008).

\begin{tabular}{ccccc}
\hline Tema & Subtema & Gestão e produção & Produção & Total \\
\hline Cadeia de suprimentos & Logística reversa & 3 & 1 & 4 \\
& Gestão ambiental & - & 1 & 1 \\
Gestão ambiental & Desenvolvimento sustentável & - & 1 & 1 \\
Desenvolvimento sustentável & - & 10 & 4 & 14 \\
Sustentabilidade & - & 3 & 2 & 4 \\
Conduta social e ambiental & - & 3 & 10 & 3 \\
Total & - & 20 & & 30 \\
\hline
\end{tabular}

Fonte: os autores.

com o meio ambiente, bem como a atuação de organizações não governamentais e, por fim, a maior sensibilidade ao tema em instituições públicas e privadas. Os editores concluem que estes fatos vêm se refletindo na produção acadêmica em diversas áreas de conhecimento (CÔRTES et al., 2006). Esta afirmação é o primeiro paralelo entre os resultados que indicam o crescimento do tema em G0 no IJOPM e a produção brasileira. Estes 30 artigos têm os resultados da análise qualitativa por afinidade, apresentado na Tabela 1. Na análise dos dados da Tabela 1, o tema Gestão Ambiental é o que apresenta maior número de publicações em ambos os periódicos. 0 tema central de G0 em SS que aparece no 1JOPM no mesmo período (2004 a 2008) é Cadeia de Suprimentos Verde. Nos periódicos brasileiros, a abordagem de SS dentro de Gestão da Cadeia de Suprimentos, é o segundo tema mais abordado, com 6 publicações.

Complementando a análise das publicações com temas de SS em periódicos brasileiros, ressalta-se que a partir de 2006 houve um crescimento da produção no tema. No caso da revista Produção, dos 10 artigos, 7 foram publicados de 2006 em diante e, no caso da Gestão \& Produção, todos os 20 artigos foram publicados a partir deste ano.

\section{Conclusões}

0 método de análise de cocitações de autores é uma técnica precisa para demonstrar a conexão intelectual entre ideias e temas de pesquisa. Através deste método, foi possível verificar que os temas em GO, atualizando Pilkington e Fitzgerald (2006), apresentam uma dinâmica que a cada período de cinco anos sofre alterações consideráveis. Pilkington e Fitzgerald (2006) analisaram um total de 829 artigos e 1.080 autores. Complementando esta base, a presente pesquisa adicionou 339 artigos e 756 autores, totalizando 1.168 artigos e 1.836 autores.

A partir desta base, a pesquisa aprofunda-se na análise dos trabalhos que referenciam a Sustentabilidade Socioambiental, identificando possíveis agrupamentos; são apresentados os principais autores citados e os artigos produzidos ao redor destes. Foram produzidas tabelas e análises com o objetivo de interpretar a produção de artigos sobre o tema no universo do IJOPM, bem como produzir um quadro comparativo com temas mais consagrados. 0 tema de Sustentabilidade Socioambiental aparece de forma realmente evolutiva e não passageira, ao contrário de outros, que aparecem com bastante densidade em determinados períodos, mas simplesmente desaparecem em períodos posteriores.

Outro fato a ser observado é que, dos artigos centrais ao longo dos três períodos, apenas um deles foi originalmente publicado no próprio IJOPM. Todos os outros artigos centrais que fizeram referências a publicações neste journal têm origem em outras publicações que não pertencem necessariamente à área de GO. A análise do perfil do que é originalmente publicado no IJOPM demonstra que os autores que publicam neste periódico estão buscando referências em outras fontes, para poderem, hipoteticamente, começar a produzir neste tema.

Na base inicial de Pilkington e Fitzgerald (2006), conforme já comentado, os artigos que remetem ao conceito de Sustentabilidade Socioambiental apresentam dois autores referenciados.

No terceiro período, adicionado pela presente pesquisa, os artigos que abordam temas relacionados à Sustentabilidade Socioambiental aparecem como o sexto mais discutido pela área, sob a nomenclatura de Green Supply Chain, e com quatro autores referenciados.

Os artigos centrais sobre o tema também apresentaram uma evolução. Inicialmente, eram muito centrados no problema e nos custos relativos ao controle e à prevenção da poluição. No segundo bloco, temas como a integração da cadeia de suprimentos (fornecedores e fabricantes), novos modelos de organização do trabalho e reciclagem passam a ser centrais, com um enfoque não somente no resultado de uma operação não aderente ao meio ambiente, 
mas também em como transformar processos de modo a minimizar os prejuizos.

A área de GO começa a incorporar temas de SS como parte da formulação de sua estratégia. Na análise da produção nacional, verificou-se também o recente interesse pelo tema. Esta constatação, o trabalho de Pilkington e Fitzgerald (2006), e somando-se os resultados da pesquisa bibliométrica utilizada para o período de 2004 a 2008, sugere que o tema Sustentabilidade Socioambiental se tornou representativo no campo de Gestão de Operações.

\section{Referências}

BARNEY, J. B. Firm resources and sustained competitive advantage. Journal of Management, v. 17, n. 1, p. 99-120, 1991. http://dx.doi.org/10.1177/014920639101700108

BARBIERI, J. C. Desenvolvimento e meio ambiente: as estratégias de mudanças da agenda 21. Petrópolis: Vozes, 1997.

BARBIERI, J. C. Gestão ambiental empresarial: conceitos, modelos e instrumentos. São Paulo: Saraiva, 2007.

BORGATTI, S. P.; EVERETT, M. G.; FREEMAN, L. C. Ucinet 6 for Windows: Software for Social Network Analysis. Harvard: Analytic Technologies, 2002.

COORDENAÇÃO DE APERFEIÇOAMENTO DE PESSOAL DE NÍVEL SUPERIOR - CAPES. Webqualis: aplicativo que permite a classificação e consulta ao Qualis das áreas, bem como a divulgação dos critérios utilizados para a classificação de periódicos. Disponível em: <http://qualis. capes.gov.br/webqualis/>. Acesso em: 13 out. 2009.

CÔRTES, M. R. et al. Co-editorial Revista Gestão e Produção, São Carlos, v. 13, n. 3, 2006.

CHARVET, F. F. et al. The intellectual Structure of Supply Chain Management: A Bibliometric Approach. Journal of Business Logistics, v. 29, n. 1, 2008. http://dx.doi. org/10.1002/j.2158-1592.2008.tb00068.x

EPELBAUM, M. Sistemas de gestão ambiental. In: VILELA JÚNIOR, A.; DEMAJOROVIC, J. (Orgs.). Modelos e ferramentas de gestão ambiental: desafios e perspectivas para as organizações. São Paulo: Senac, 2006.

FERDOWS, K.; DE MEYER, A. Lasting Improvements in Manufacturing Performance: In search of a New Theory. Journal of Operations Management, v. 9, n. 2, p. 168-184, 1990. http://dx.doi.org/10.1016/02726963(90)90094-T

FLORIDA, R. Regional Creative Destruction: Production Organization, Globalization, and the Economic Transformation of the Midwest. Economic Geography, v. $72, \quad$ n. 3, p. 314-334, 1996. http://dx.doi. org/10.2307/144403

GEFFEN, C.; ROTHENBERG, S. Suppliers and Environmental Innovation: The Automotive Paint Process. International Journal of Operations and Production Management, v. 20, n. 20, p. 166-186, 2000. http://dx.doi. org/10.1108/01443570010304242

$\mathrm{GOH}, \quad \mathrm{C}-\mathrm{H}$. et al. Evaluating and Classifying POM Journals. Journal of Operations Management, n. 15, p. 123-138, 1997. http://dx.doi.org/10.1016/S02726963(96)00102-7
GRANT, R. The resource-based theory of competitive advantage: implications for strategy formulation. California Management Review, v. 33, n. 3, p. 114-135, 1991.

HAIR JUNIOR, J. F. et al. Análise Multivariada de Dados. 5. ed. Porto Alegre: Bookman, 2005.

HART, S. A Natural-Resource-Based View of the Firm. Academy of Management Review, v. 20, n. 4, p. 986-1014, 1995.

HAYES, R. H.; WHEELWRIGHT, S. C. Restoring our Competitive Edge: Competing Through Manufacturing. New York: Wiley, 1984.

HÖJER, et al. Scenarios in selected tools for environmental systems analysis. Journal of Cleaner Production, v. 16, p.1958-1970, 2008. http://dx.doi.org/10.1016/j. jclepro.2008.01.008

HUNT, J.; RAMAN, S. Regulation matters: global environmental discourse and business response. In: FINEMAN, S. (Ed.). The Business of Greening. London: Routledge, 2000. p. 114-133.

JABBOUR, C. J. C.; SANTOS, F. C. A. Evolução da gestão ambiental na empresa: uma taxonomia integrada à gestão da produção e de recursos humanos. Gestão \& Produção, v. 13, n. 3, p. 435-448, 2006. Disponível em: $<$ http://www.scielo.br/scielo>.

KLEINDORFER, P. R.; SINGHAL, K.; WASSENHOVE, L. N. V. Sustainable Operations Management. Production and Operations Management, v. 14, n. 4, p. 482-484, 2005. http://dx.doi.org/10.1111/j.1937-5956.2005.tb00235.x

KING, A. Organizational response to environmental regulation: punctuated change or autogenesis? Business Strategy and the Environment, v. 9, n. 4, p. 224-238, 2000. http://dx.doi. org/10.1002/1099-0836(200007/08)9:4\%3C224::AIDBSE249\%3E3.0.CO;2-X

KING, A.;LENOX, M. Doesit Really Pay to be Green? An Empirical Study of Firm Environmental and Financial Performance. Journal of Industry Ecology, v. 5, n.1, p. 105-116, 2001. http://dx.doi.org/10.1162/108819801753358526

MIN, H.; GALLE, W. Green Purchasing Strategies: Trends and Implications International Journal of Purchasing and Materials, Summer, v. 33, n. 3, p. 10-17, 1997.

MILLER, J. G.; ROTH, A. A Taxonomy of Manufacturing Strategies. Management Science, v. 40, n. 3, 1994. http://dx.doi.org/10.1287/mnsc.40.3.285

OKUBO, Y. Bibliometric Indicators and analysis of research systems: methods and examples. Paris: OECD, 1997. 69 p. (STI Working Papers, 1997/1).

OLIVEIRA, J. G.; BORGES, F. H.; JABBOUR, C. C. 0 Impacto Competitivo da Estratégia Ambiental: uma abordagem teórica. In: Simpósio Internacional de Gestão Ambiental e Saúde - SIGAS, 1., 2005, São Paulo. Anais... São Paulo: SENAC, 2005.

PICKMAN, H. A. The effect of environmental regulation on environmental innovation. Business Strategy and the Environment, v. 8, n. 3, p. 189-196, 1998.

PILKINGTON, A.; FITZGERALD, R. Operations Management Themes, conceptsand relationships: a forward retrospective of 1JOPM. International Journal of Operations \& Product, Management, vol. 26, n. 11, p. 1255-1275, 2006. http:// dx.doi.org/10.1108/01443570610705854

PILKINGTON, A.; MEREDITH, J. The Evolution of the intellectual structure of operations management - 1980-2006: A citation/co-citation analysis. Journal of Operations Management, v. 27, p. 185-202, 2009. http:// dx.doi.org/10.1016/j.jom.2008.08.001 
PORTER, M. Competitive strategy: techniques for analyzing industries and competitors. New York: Free Press, 1980.

PORTER, M.; VAN DER LINDE, C. Green and Competitive: Ending the Stalemate. Harvard Business Review, v. 73, n. 5, p. 120-134, 1995.

RAMOS-RODRIGUEZ, A. R.; RUIZ-NAVARRO, J., Changes in the intellectual structure of strategic management research: a bibliometric study of the Strategic Management Journal, 1980-2000. Strategic Management Journal, v. 25, n. 10, p. 981-1004, 2004. http://dx.doi. $\operatorname{org} / 10.1002 /$ smj.397

REVISTA GESTÃO \& PRODUÇÃO. São Carlos: Universidade Federal de São Carlos, 1994-. Disponível em <http:// www.scielo.br/scielo.php?script=sci_issues $\&$ pid $=0104$ 530X\&lng=pt\&nrm=iso>. Acesso em: 13 out. 2009.

REVISTA PRODUÇÃO. São Paulo: Associação Brasileira de Engenharia de Produção (ABEPRO), Departamento de Engenharia de Produção da Escola Politécnica da Universidade deSão Paulo (PRO/EPUSP), 1990-.Disponível em: $\quad<$ http://www.scielo.br/scielo.php?script=sci_ issues \&pid $=0103-6513 \& \operatorname{lng}=p t \& n r m=i s o>$. Acesso em: 13 out. 2009.

SACHS, 1. Estratégias de transição para o século XXI: desenvolvimento e meio ambiente. São Paulo: Studio Nobel, Fundação de Desenvolvimento Administrativo FUNDAP, 1993.

SCHAEFER, A. Corporate greening and changing regulatory regimes: the UK water industry. Business Strategy and the Environment, v. 18, p. 320-333, 2009. Disponivel em: <www.interscience.wiley.com>. http://dx.doi. $\operatorname{org} / 10.1002 /$ bse.591

SCHAEFER, A., HARVEY, B. Agents of change in corporate greening: case studies in water and electricity utilities. In: FINEMAN, S. (Ed.). The Business of Greening. London: Routledge, 2000. p. 169-188.

SCHILDT, H. A. SITKIS: Software for Bibliometric Data Management and Analysis v0.6.1. Helsinki: Institute of Strategy and International Business, 2002. Disponivel em: <www.hut.fi/ hschildt/sitkis>.
SEIFFERT, M. E. B. Sistemas de gestão ambiental (ISO 14001) e saúde e segurança ocupacional (OHSAS 18001): vantagens da implantação integrada. São Paulo: Atlas. 2008.

SIMON, D. Dilemmas of development and the environment in a globalizing world: theory, policy and praxis. Progress in Development Studies, Londres, v. 3, n. 1, p. 5-41, 2003. http://dx.doi.org/10.1191/1464993403ps048ra

SILVA, E. M.; SANTOS, F. C. A. Estratégia de produção, melhores práticas e medição de desempenho: revisão, lacunas e planejamento para futuras pesquisas. Revista Gestão Industrial, Ponta Grossa, v. 2, n. 1, p. 64-74, 2006.

SKINNER, W. Manufacturing - missing link in corporate strategy. Harvard Business Review, v. 47, n. 3, p. 136-145, 1969.

SMALL, H. G. Co-citation in the Scientific Literature: A New Measure of the Relationship Between Documents. Journal of American Society for Information Science, v. 24 , n. 4 , p. 265-26, 1973. http://dx.doi.org/10.1002/ asi.4630240406

SMITH, M.; CROTTY, J. Environmental regulation and innovation driving ecological design in the UK automotive industry. Business Strategy and the Environment, v. 17, n. 6, p. 341-349, 2006. Disponível em: <www.interscience. wiley.com>. http://dx.doi.org/10.1002/bse.550

SWAMIDASS, P. M.; NEWELL W. T. Manufacturing Strategy, Environmental Uncertainty and Performance: a path and analytic model. Management Science, v. 33, n. 4, p. 509-524, 1987. http://dx.doi.org/10.1287/ mnsc.33.4.509

VILELA JUNIOR, A.; DEMAJOROVIC, J. Modelos e ferramentas de gestão ambiental: desafios e perspectivas para as organizações. São Paulo: Senac, 2006.

WERNERFELT, B. A resource-based view of the firm. Strategic Management Journal, v. 5, n. 2, p. 171-180, 1984. http://dx.doi.org/10.1002/smj.4250050207

\title{
Environmental sustainability: a bibliometric study of its evolution in operations management
}

\begin{abstract}
Sustainability and sustainable development have offered a great challenge for organizations, governments and society, in order to make the new paradigm effective in managerial and operational practices. In the field of Operations Management (OM), researchers and organizations have been trying to develop and incorporate new concepts, models and tools in an effort to adjust their management procedures to this new paradigm. This article presents a bibliometric study and proposes to investigate the frequency of the sustainability theme in academic research, within the scope of OM, analyzing articles published in the International Journal of Operations \& Production Management and making a parallel with relevant OM Brazilian Journals. Main results presented the evolution of sustainability in Operations Management and the identified themes within. The main conclusion, ranking its themes by co-citation, is that sustainability was identified as an emerging theme and it is closely related to Manufacturing Strategy discussions in the OM field.
\end{abstract}

\section{Keywords}

Sustainability. Operations management. Bibliometric study. Sustainable resource view. Green supply chain. 